\title{
SAR Image Processing Algorithms Based on the Ambiguity Function.
}

\author{
Ana B. Ramirez, Ivan J. Rivera, Domingo Rodriguez \\ Automated Information Processing Laboratory \\ Electrical and Computer Engineering Department \\ University of Puerto Rico \\ Mayaguez, PR 00681-4092 \\ e-mail:\{ana.ramirez, ivan.rivera, domingo\}@ece.uprm.edu
}

\begin{abstract}
This work focuses on hardware implementations of the ambiguity function defined in terms of signal operators for Synthetic Aperture Radar (SAR) sensor image formation applications. Due to the large quantity of data associated with SAR imaging, basic SAR operations demand high computational cost and are difficult to readily implement on existing commercial digital signal processing (DSP) units. In order to contribute to resolve this problem a search for new efficient algorithm variants in under way compute operations such as discrete Fourier transforms, discrete convolutions, discrete correlations, and Hadamard products of complex signals. These operations are commonly used in the computation of the discrete ambiguity function.

Time-frequency tools have been the object of many different studies in SAR image formation operations. The principle behind SAR image formation is recovering a desired reflectivity density function through an inverse convolution process on the raw data generation. It is this particular fact that makes the ambiguity function so important in SAR image formation since it serves to model the impulse response function or point spread function of the SAR system itself.

Algorithms for computing the ambiguity designed for this work are being developed in MATLAB and implemented the TMS320C6713 digital signal processor (DSP) unit. The implementation methodology is based on hardware/software algorithm co-design techniques, taking into consideration software and hardware characteristics such as memory cache overflows, twiddle or phase factor pre-computations, extended memory addressing schemes, stride permutation implementations, pipelining schemes, multiply-add DSP architectures, multiple concurrent memory accessing schemes, and overall latency response. ${ }^{1}$
\end{abstract}

\section{INTRODUCTION}

A Digital Signal Processors system is composed by a combination of hardware and software components such us different kinds of memory modules, I/O interfaces, development environments, etc. Is essential in hardware and software co-design to select the appropriate hardware components to the algorithms to be implemented, choose the most appropriate transfer data mode and also know the memory requirements. Depending of the application each issue can be selected. Digital Signal Processing applications such as Synthetic Aperture Radar are demanding high processing power because of the large quantity data generated for the radar system.

\footnotetext{
${ }^{1}$ Partially supported by NSF Grant No. CNSO424546
}

Due to the low quantity of memory that is available in a Digital Signal Processor, the hardware implementation implies takes into account characteristics of data transfer between the DSP and external memory or a host PC. Also, it is necessary has pre-computations of the twiddle factors stored in memory which will be used to compute rapid algorithms of the Discrete Fourier Transform.

\section{Signal Algebra Concepts}

The Cross-Ambiguity Function $A_{\{p, q\}}$ of two signals $p(t)$ and $q(t)$ that belong to the Hilbert space $L^{2}(R)$ of all the complex-value functions of the real-time variable $t$ is defined as Equation 2.

In a formal way, let, $p(t)$ and $q(t)$ arbitrary signals and $t \in R$, the cross-ambiguity function signal representation of $p$ and $q$ is defined as follows:

$$
\begin{gathered}
A: L^{2}(R) \times L^{2}(R) \rightarrow L^{2}(R \times R) \\
(p, q) \mapsto A_{\{p, q\}}
\end{gathered}
$$

where,

$$
A_{\{p, q\}}(\tau, f)=\int_{-\infty}^{\infty} p(t+\tau) q^{*}(t) e^{-j 2 \pi f t} d t
$$

Using representation theory of groups, a discrete formulation of the Ambiguity Function [1] can be expressed in terms of two discrete, $\mathrm{N}$-periodic signals $p$ and $q$ :

$$
\begin{gathered}
A: L^{2}\left(Z_{N}\right) \times L^{2}\left(Z_{N}\right) \rightarrow L^{2}\left(Z_{N} \times Z_{N}\right) \\
(p, q) \mapsto A_{\{p, q\}}
\end{gathered}
$$

where,

$$
A_{\{p, q\}}[m, k]=\sum_{n \in Z_{N}} p[n] q^{*}\left[\langle n+m\rangle_{N}\right] e^{-j \frac{2 \pi k n}{N}}
$$




\section{SAR BASIC CONCEPTS}

The basic idea is to use the Ambiguity Function as Point Spread Function of the radar system. Taking the transmitted $S_{T}[n]$ and the received $S_{R}[n]$ signals and computing the Ambiguity Function is possible to get the system impulse response, which is used for synthetic aperture radar image formation, that is the inverse process of the raw data or level zero data generation. A scheme for the generation of the impulse response function using the $\mathrm{AF}$ is presented in Fig. 1, where $m$ is the time delay and $k$ is the frequency shift of the received signal $S_{R}[n]$. In image formation, basic geometric configuration uses the spatial coordinates $(x, r)$ that are referred to the azimuth and range coordinates respectively and can be related with the coordinates $(m, k)$.

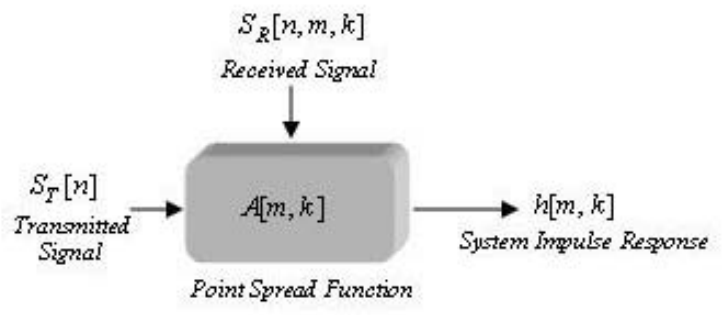

Fig. 1. Point Spread Function

The raw data generation consists in compute the 2-D cyclic convolution between the impulse response function and the reflectivity function where the reflectivity function is the representation of the scene and the SAR imaging problem consists in design an adequate system impulse response so that the best estimate of the representation of the scene can be obtained using the raw data. It is schematized on Fig. 2. Based on those concepts, the reflectivity function can be obtained as the inverse process of the raw data generation that can be reduced to a $2-\mathrm{D}$ cyclic convolution (See Fig. 3).

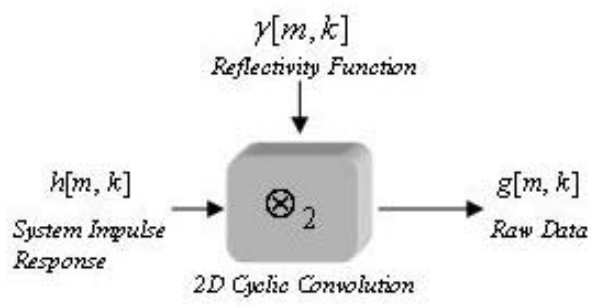

Fig. 2. Raw Data Generation

\section{HARDWARE REQUIREMENTS}

The complex data is organized to have real part follow of the imaginary part and the organized data is stored on external memory or a PC host computer before processing.

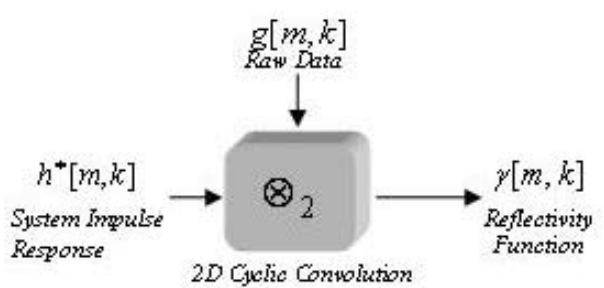

Fig. 3. Reflectivity Function Estimation

The hardware implementation is made on a Digital Signal Processor TMS320C6713 of Texas Instruments, which has functional units that can perform floating point operations for higher precision. There is not enough memory included with those processors but it provides an interface to external memory. In Fig 4. is presented the interface provided by Texas Instruments processor to access data stored in external memory or a host computer in real time without halt the processor and the functions related to each part of the hardware system. The data is transferred using the Real-Time Data Exchange (RTDX) tool.

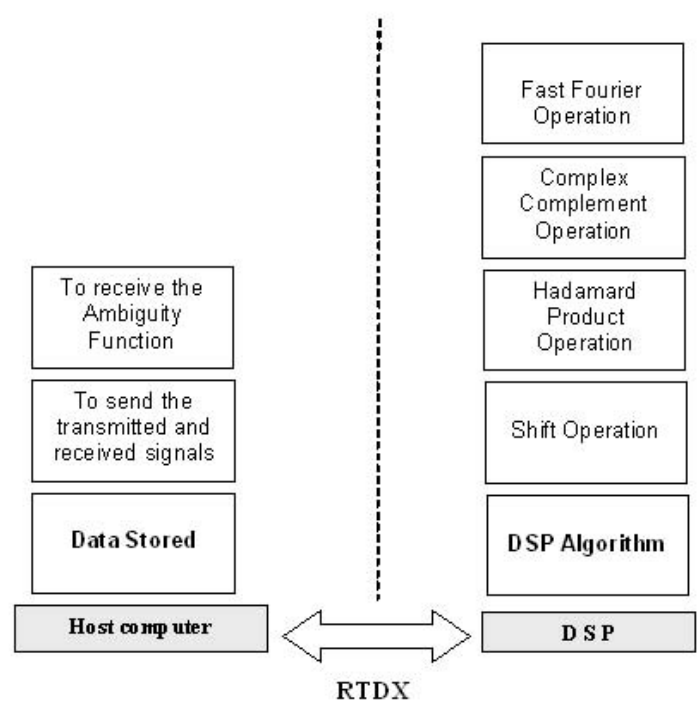

Fig. 4. Real-Time Data Exchange

The RTDX tool can be used in both directions: from the DSP to the host computer or from the host computer to the DSP, without interfere with the DSP application.

\section{Ambiguity Function Computation}

A radar system sends pulses $S_{T}[n]$ to illuminate an area on the ground and receives backscattered signals from each 
target $S_{R}[n]$ in the illuminated area. An objective returns a signal that is the same as the sent signal but delayed in time by a quantity $m$ and shifted in frequency by a quantity $k$. The Ambiguity Function can be computed according to the Equation 4.

$$
A_{\left\{S_{T}, S_{R}\right\}}[m, k]=\sum_{n \in Z_{n}} S_{T}[n] S_{R}^{*}[n+m] e^{(-j 2 \pi k n / N)}
$$

The hardware implementation of the Ambiguity Function is based on the algebra of operators using the Transform Method. The Ambiguity Function can be seen as the Discrete Fourier Transform of the product between the transmitted signal and the received signal shifted by $m$. The stored transmitted and received signals are sent to the DSP via RTDX. The target or DSP has different tasks: To make the shift to the received signal, then the Hadamard product between the transmitted and the received shifted signal that has been conjugated and finally the FFT of the result.

The complete process used to get the ambiguity function in operator terms using the Transform Method is shown in Fig 5. Once these processes are made, the results are sent to the host PC via RTDX. Each result that is sent to the PC corresponds to a row of the ambiguity matrix.

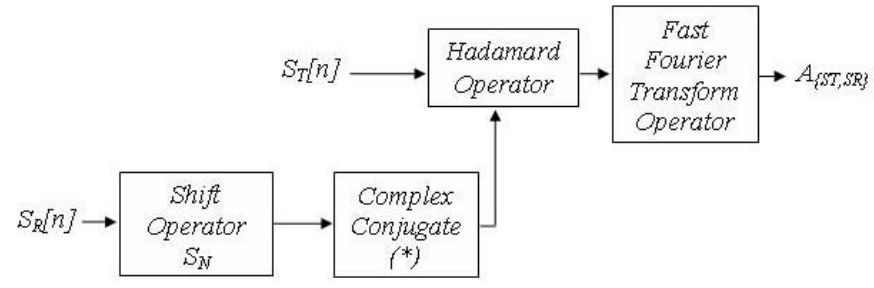

Fig. 5. Ambiguity Function Computation Method

An example of the Ambiguity Function obtained using the Real-Time Data Exchange for two real Chirp signals $p[n]$ and $q[n]$ of 1024 points in double precision, that have been used to simulate the transmitted and received signals is shown in Fig 6.

\section{ANALYSIS OF PERFORMANCE}

Different factors can contribute to analyze the execution time of the method proposed such us the algorithm implemented, the selected DSP, the selected data type, the transfer data mode and the used software to interface the host PC with the DSP.

Analysis of execution time: One of the measures to determine the system performance is the execution timing of the procedure. The process that spends more time is the data transfer between the external memory and the DSP.

Using Matlab as simulation enviroment to interface the host PC and the DSP for data exchange, the execution times shown in the table 1 for the procedures enclosed in this application.

Different Hardware-Software Co-design tools are being used to prove performance analisys for SAR image processing

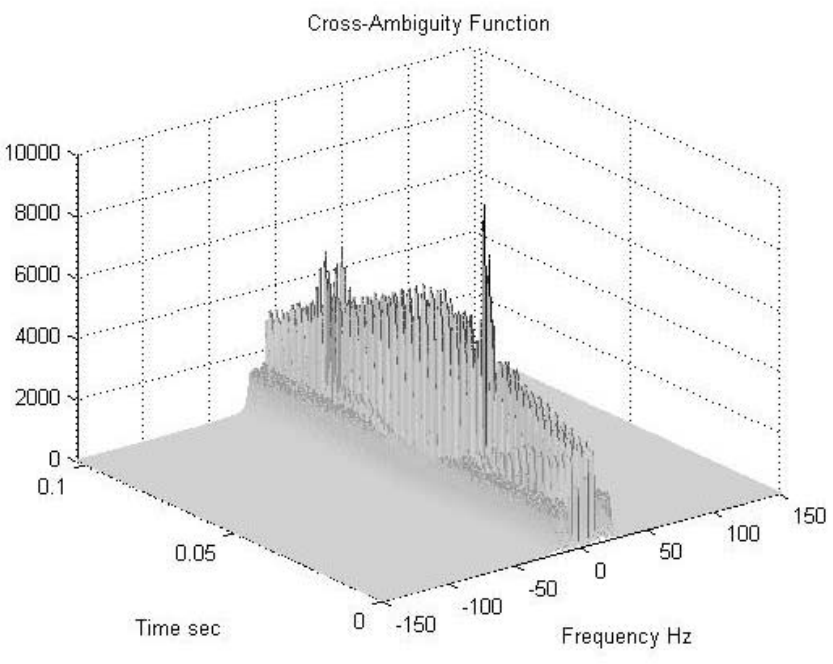

Fig. 6. Cross-Ambiguity Function Computation

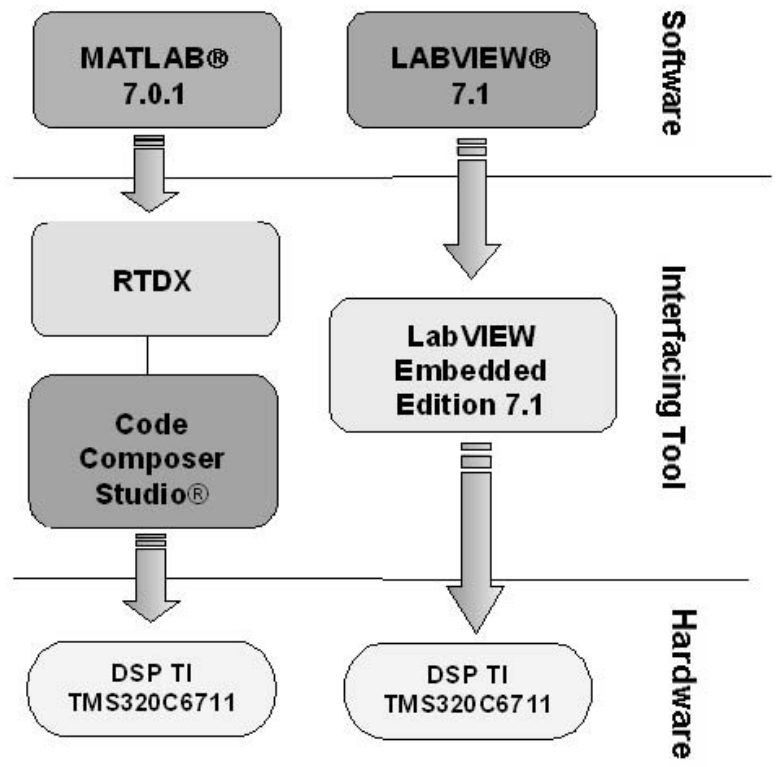

Fig. 7. Hardware Software Co-design Tools

algorithms. In Fig. 7 is presented two schemes of hardwaresoftware tools based on DSP modules and the first graphic represents the scheme used along this work.

\section{CONCLUSIONS}

Incorporating memory access in a hardware implementation of a SAR real time application allows to handle the limited memory capacity in digital signal processors and it is related with parallel processing. In future works a multiprocessors 
TABLE I

EXECUTION Times OF THE DSP TO PC DATA TRANSFER

\begin{tabular}{|c|c|}
\hline $\begin{array}{c}\text { No. of points } \\
\text { of a signal } \\
\text { (Double Datatype) }\end{array}$ & $\begin{array}{c}\text { DSP to PC } \\
\text { Transfer } \\
\text { (Seconds) }\end{array}$ \\
\hline 2048 & 6 \\
4096 & 10.2 \\
8192 & 26.8 \\
16384 & 54 \\
\hline
\end{tabular}

system can improve hardware implementation of SAR image processing algorithms using real-time data exchange between DSP's and memory modules.

\section{REFERENCES}

[1] M.S. Richman, T.W. Parks and R.G. shenoy Discrete-Time, DiscreteFrequency, Time-Frequency Representations. IEEE, 1995.

[2] L. Auslander and R. Tolimieri, Characterizing the Radar Ambiguity Functions. IEEE Transactions on Information theory, Vol 30, No.6, 1984.

[3] L. Bierens and E. Deprettere, Engineering Multirate Convolutions For Radar Imaging. IEEE, 1996.

[4] L. Ming, W. Yan, W. Shun-jun and Y. Wei-ming Implementation of an Parallel Signal Processing System for All-purpose Radar. In ICSP Proc. IEEE, 2002.

[5] G. Franceschetti and G. Schirinzi A SAR Processor Based on TwoDimensional FFT Codes. IEEE Transactions on Aerospace and Electronic Systems, Vol 26, No.2, 1990.

[6] T. J. Abatzoglou and G. O. GheenRange, Radial Velocity and Acceleration MLE Using Radar LFM Pulse Train. IEEE Transactions on Aerospace and Electronic Systems, Vol 34, No.4, 1998.

[7] S. Stein Algorithms for Ambiguity Function. IEEE, 1981. 